\title{
Modeling Inaccurate Perception: Desynchronization Issues of a Chaotic Pattern Recognition Neural Network
}

\author{
Dragos Calitoiu, B. John Oommen, and Dorin Nusbaumm \\ Carleton University, Ottawa, K1S 5B6, Canada
}

\begin{abstract}
The usual goal of modeling natural and artificial perception involves determining how a system can extract the object that it perceives from an image which is noisy. The "inverse" of this problem is one of modeling how even a clear image can be perceived to be blurred in certain contexts. We propose a chaotic model of Pattern Recognition (PR) for the theory of "blurring". The paper, which is an extension to a Companion paper [3] demonstrates how one can model blurring from the view point of a chaotic PR system. Unlike the Companion paper in which the chaotic PR system extracts the pattern from the input, this paper shows that the perception can be "blurred" if the dynamics of the chaotic system are modified. We thus propose a formal model, the $\mathrm{Mb}-\mathrm{AdNN}$, and present a rigorous analysis using the Routh-Hurwitz criterion and Lyapunov exponents. We also demonstrate, experimentally, the validity of our model by using a numeral dataset.
\end{abstract}

\section{Introduction}

Sensations can be explained as simple experiences that are caused by physical stimuli. Perceptions are processes by which the brain organizes and interprets sensations [2]. What we know about the world depends on the information gathered by our senses and channeled into our brains. Our brain organizes sensations in such a way that enables us to locate and recognize objects in the environment. However, we are capable of perceiving only those objects to which we devote attention. Perception is not entirely innate, we can learn to perceive [10]. In this paper we consider the "inverse" of the perception problem, namely that of modeling how a clear image can be perceived to be blurred in certain contexts. We propose a chaotic model of PR for the theory of "blurring".

\subsection{Perceptual Organization and Inaccurate Perception}

Sensations are caused by the sensory system, that is stimulated by objects and by events in the environment. The brain organizes sensations in such a way that it can locate and recognize objects and events in the environment, and then detect an activity related to these objects. Understanding perceptual organization is a matter of understanding how one perceives form, depth, and motion. Gestalt 
psychologists argue that the key to understanding perception is to identify the relations between various parts of a stimulus as the relationship between the lines of a triangle.

Perception, in itself, is not a reception of what is received. It involves a processing of what is received, and an activity of analysis, synthesis and generalization. The essence of perception is the continuous exploration of the objects. This exploration can become, under certain conditions, disorganized and incoherent, which can lead to a high level of perturbation of the operations involved in perception. This paper intends to model the perturbation of perception.

Perception is a conscious process, which is oriented and organized. It involves many actions, such as measuring, decomposing, recomposing, grouping, classifying, transforming and modeling of the objects . As an active mechanism, perception is created, corrected, and verified by actions. When an action, involving objects is limited, the perception is poor, incomplete or erroneous.

The brain is a system with many complex functions and creating an artificial model, which can handle all the functions associated with the brain, is very difficult. Often, the idea of optimizing one function leads to a decline in importance of other functions involved in the overall activity of the model. In this research we study the concept of perception and its "inverse", which is denoted here as blurring or inaccurate perception. We would like to understand the function of such an artificial recognition system by modelling inaccurate perception. We accomplish this by exploring a model, used for Pattern Recognition (PR), which was presented in the Companion paper [3]. This model can also be used to analyze the effects of anomalies to the process of perception.

To understand this, consider a real life analogy. There are two types of problems which affect the process of perception in visual recognition:

(i) Issues regarding the stimulus, which in this case involve the resolution of the image, and

(ii) Issues regarding the recognition system itself. Three reasons why vision can be blurred are:

- the eye itself has a disease that affects the reception of a stimulus (e.g. by decreasing the visual acuity, for example by myopia, hyperopia, astigmatism);

- the optical nerve is unhealthy, thus affecting the transmission of a stimulus;

- the visual cortex is unhealthy, thus affecting the processing of a stimulus.

The second class of problems, which are related to the quality of a recognition system, have not been studied extensively in the modelling of artificial perception systems. This leads us, quite naturally, to the "inverse" problem, which consists of the perturbation of recognition. This is unlike previous studies, which primarily address the degradation of the quality of the stimulus (e.g., by adding noise, decreasing the resolution, decreasing the number of bits per pixel). In this paper we propose a new approach for such modeling, where the ability of a system can be modified by changing its dynamics without changing the input (i.e., the stimulus). We will try to provide an answer to a general question: "Is there a chaotic model for why the system inaccurately processes knowledge even if the stimulus is perfect?". 


\subsection{Artificial Perception Models Involved in Recognition}

The four best approaches for modeling artificial PR systems are: template matching, statistical classification, syntactic or structural recognition, and Artificial Neural Networks (ANNs) 6, 8, 11, 13. Some popular models of ANNs have been shown to have associative memory and learning [5, 9, 12. The learning process involves updating the network architecture and modifying the weights between the neurons so that the network can efficiently perform a specific classification or clustering task.

An associative memory permits its user to specify part of a pattern or a key, and to then retrieve the values associated with that pattern. This ability of the brain to "jump" from one memory state to another in the absence of a stimulus is one of the hallmarks of the brain, and this phenomenon we want to emulate.

The evidence that indicates the possible relevance of chaos to brain functions was first obtained by Freeman 7 . through his clinical work on the large-scale collective behavior of neurons in the perception of olfactory stimuli. Thus, mimicing this identification on a neural network can lead to a new model of pattern recognition, which is the goal of this research endeavor.

A Chaotic Neural Network (CNN) with non-fixed weights of the connections between neurons can reside in one of the infinite number of possible states that are allowed by the functions of the network. In cases where a memorized pattern is given as the input to the network, we want the network to resonate with that pattern, generating that pattern with, hopefully, small periodicity, where the actual period of resonance is not of critical importance. Between two consecutive appearances of the memorized pattern, we would like the network to be in one of the non-memorized infinite number of states with a high probability, and in the memorized states with an arbitrary small probability. All this is achieved using our NN, the Modified Adachi Neural Network (M-AdNN), introduced in [3. How to "juggle" between chaos and periodicity is, indeed, an art, and this is what we attempt to control.

The sympathetic resonance1 1 with the memorized pattern given as input, and the transition through several states from the infinite set of possible states (even when the memorized pattern is inserted as the input) represent the difference between this kind of PR and the classical types which correspond to the strategies associated with syntactical or statistical pattern recognition.

Classical neural computation does not include Chaotic Neural Networks. By modifying the M-AdNN model, proposed in [3, we formulate a model for the foundation of inaccurate perception, caused by the properties of the system and not by the quality of the stimulus. To our knowledge, such a phenomenon has not been proposed in previous models. The reason for excluding such a phenomenon in the modeling is not due to the lack of evidence of this property in human

${ }^{1}$ The term "sympathetic resonance" is used for lack of a better one. Quite simply, all we require is that the trained pattern periodically surfaces as the output of the CNN. 
perception; rather it is due to a lack of a mechanism that can "implement" it. Thus, in essence, the contribution of our paper is a formal chaotic model for both perception and blurring, which has been experimentally verified.

\section{Adachi's Model for Neural Networks: The AdNN}

The AdNN, which actually is a Hopfield-like model, is composed of $N$ neurons, topologically arranged as a completely connected graph. It is modelled as a dynamical associative memory, by means of the following equations relating internal states $\eta_{i}(t)$ and $\xi_{i}(y), i=1 . . N$, and the output $x_{i}(t)$ as:

$$
\begin{aligned}
& x_{i}(t+1)=f\left(\eta_{i}(t+1)+\xi_{i}(t+1)\right), \\
& \eta_{i}(t+1)=k_{f} \eta_{i}(t)+\sum_{j=1}^{N} w_{i j} x_{j}(t), \\
& \xi_{i}(t+1)=k_{r} \xi_{i}(t)-\alpha x_{i}(t)+a_{i} .
\end{aligned}
$$

In the above, $x_{i}(t)$ is the output of the neuron $i$ which has an analog value in $[0,1]$ at the discrete time " $t$ ", and $f$ is the logistic function with the steepness parameter $\varepsilon$ satisfying $f(y)=1 /(1+\exp (-y / \varepsilon))$. Additionally, $k_{f}$ and $k_{r}$ are the decay parameters for the feedback inputs and the refractoriness, respectively, $w_{i j}$ are the synaptic weights to the $i^{\text {th }}$ constituent neuron from the $j^{\text {th }}$ constituent neuron, and $a_{i}$ denotes the temporally constant external inputs to the $i^{t h}$ neuron. Finally, the feedback interconnections are determined according to the following symmetric auto-associative matrix of the $p$ stored patterns as in: $w_{i j}=\frac{1}{p} \sum_{s=1}^{p}\left(2 x_{i}^{s}-1\right)\left(2 x_{j}^{s}-1\right)$ where $x_{i}^{s}$ is the $i^{t h}$ component of the $s^{t h}$ stored pattern.

In his instantiation, Adachi set $N=100, p=4, k_{f}=0.2$ and $k_{r}=0.9$.

Adachi et al. also investigated the dynamics of the associative network with external stimulations corresponding to one of the stored patterns when the external inputs are applied, and they did this by increasing their bias terms, $a_{i}$, as in: $a_{i}=2+6 x_{i}^{s}$. The rationale for this can be seen in [1, 3, 4,

\section{$3 \quad$ PR Using Chaotic Neural Networks : The M-AdNN}

In 3] we proposed a model of CNNs which modified the AdNN as presented below. In each case we give a brief rationale for the modification.

1. The M-AdNN has two global states used for all neurons, which are $\eta(t)$ and $\xi(t)$ obeying:

$$
\begin{aligned}
& x_{i}(t+1)=f\left(\eta_{i}(t+1)+\xi_{i}(t+1)\right), \\
& \eta_{i}(t+1)=k_{f} \eta(t)+\sum_{j=1}^{N} w_{i j} x_{j}(t), \\
& \xi_{i}(t+1)=k_{r} \xi(t)-\alpha x(t)+a_{i} .
\end{aligned}
$$


After time $t+1$, the global states are updated with the values of $\eta_{N}(t+1)$ and $\xi_{N}(t+1)$ as: $\eta(t+1)=\eta_{N}(t+1)$ and $\xi(t+1)=\xi_{N}(t+1)$.

Rationale: At every time instant, when we compute a new internal state, we only use the contents of the memory from the internal state for neuron $N$. This is in contrast to the AdNN in which the updating at time $t+1$ uses the internal state values of all the neurons at time $t$.

2. The weight assignment rule for the M-AdNN is the classical variant: $w_{i j}=$ $\frac{1}{p} \sum_{s=1}^{p}\left(x_{i}^{s}\right)\left(x_{j}^{s}\right)$. This again, is in contrast to the AdNN which uses $w_{i j}=$ $\frac{1}{p} \sum_{s=1}^{p}\left(2 x_{i}^{s}-1\right)\left(2 x_{j}^{s}-1\right)$.

Rationale: We believe that the duration of the transitory process will be short if the level of chaos is low. A simple way to construct hyperchaos with all Lyapunov positive exponents is to couple $N$ chaotic neurons, and to set the couplings between the neurons to be small when compared with their self-feedbacks, i.e $w_{i i} \gg w_{i j}(i \neq j)$. For the M-AdNN, the value of $w_{i, i}$ will be zero in the identical setting. Clearly, the M-AdNN has a smaller self-feedback effect than the AdNN.

3. The external inputs are applied, in the M-AdNN, by increasing the biases, $a_{i}$, from 0 to unity whenever $x_{i}^{s}=1$, keeping the other biases to be 0 whenever $x_{i}^{s}=0$.

Rationale: In our case $a_{i}=x_{i}^{s}$, as opposed to the AdNN in which $a_{i}=$ $2+6 x_{i}^{s}$. In the case of the latter, the range of inputs is $[2,8]$ unlike the MAdCNN for which the range is $[0,1]$. Thus, it is expected that the M-AdNN will be more "receptive" to external inputs, and therefore lead to a superior PR system.

The M-AdCNN has the following properties:

Theorem 1: The M-AdNN described by Equations (1) is locally unstable whenever $N^{1 / 2}>\max \left(1 / k_{r}, 1 / k_{f}\right)$, as demonstrated by its Lyapunov spectrum.

Theorem 2: As seen by a Routh-Hurwitz analysis, the necessary conditions for the M-AdNN described by Equations (1) to be locally unstable, are that $k_{f}>0$ and $k_{r}>0$.

The proofs for these theorems can be found in the Companion paper [3].

\subsection{Designing Chaotic PR Systems}

As opposed to the accepted models of statistical, syntactical and structural PR, we do not foresee chaotic PR systems to report the identity of testing pattern with a "proclamation" of the class of the tested pattern. Instead, what we are attempting to achieve is to have the chaotic PR system continuously demonstrate chaos as long as there is no pattern to be recognized or whenever a pattern that is not to be recognized is presented. But, when a pattern, which is to be recognized, is presented to the system, we would like the proclamation of the 
identity to be made by requiring the chaos level to decrease significantly, and the system to simultaneously possess a strong periodic component, which we refer to as "sympathetic resonance".

In our testing [3], only one pattern is recognized. If the testing pattern is close enough to two or more "training" patterns, then both (all) may be periodically repeated. This is the rationale for the array count which records the periodicity of all the trained patterns in the output. The procedure for the PR system is in [4.

Instead of invoking Fourier analysis of the output spectrum, we maintained an array of output signals and tested the sequence for periodicity in the time domain.

\section{A Chaotic NN for Inaccurate Perception: The Mb-AdNN}

The rationale for transforming the AdNN into the M-AdNN was essentially one of forcing the former system into chaos, and at the same time attempting to demonstrate a periodic behaviour if we needed to. When it concerns inaccurate perception, it appears as if we have to strike a happy medium between the AdNN and the M-AdNN. For the first part, unlike the AdNN, we would like the new system to demonstrate chaos. But, on the other hand, unlike M-AdNN, we don't want the system to be "all too periodic" (i.e., or stable) if the trained non-noisy patterns are presented, because we are, indeed, attempting to model the lack of perception or "blurring".

We shall show how this can be achieved by modifying the dynamics of the control system so as to force the number of non-zero eigenvalues to be $2 m$, where $m$ is a "parameter" of the model. In each case, we show how we modify the $\mathrm{AdNN}$ (or the M-AdNN) to yield the Mb-AdNN and present the rationale for the modification.

1. The Mb-AdNN has two global states used for the first $m$ neurons, which are $\eta(t)$ and $\xi(t)$ obeying:

$x_{i}(t+1)=f\left(\eta_{i}(t+1)+\xi_{i}(t+1)\right)$,

$\eta_{i}(t+1)=k_{f} \eta(t)+\sum_{j=1}^{N} w_{i j} x_{j}(t)$, for $i \leq m$,

$\eta_{i}(t+1)=k_{f} \eta_{i}(t)+\sum_{j=1}^{N} w_{i j} x_{j}(t)$, for $i>m$,

$\xi_{i}(t+1)=k_{r} \xi(t)-\alpha x(t)+a_{i}$, for $i \leq m$,

$\xi_{i}(t+1)=k_{r} \xi_{i}(t)-\alpha x(t)+a_{i}$, for $i>m$.

After time $t+1$, the global states are updated with the values of $\eta_{m}(t+1)$ and $\xi_{m}(t+1)$ as: $\eta(t+1)=\eta_{m}(t+1)$ and $\xi(t+1)=\xi_{m}(t+1)$.

Rationale: Unlike in the AdNN, at every time instant, when we compute a new internal state vector, we shall use the contents of the memory from $m$ other internal states. Our current model lies in between the two extremes, namely (a) The AdNN model, where the updating at time $t+1$ uses the 
internal state values of all the neurons at time $t$, and (b) The M-AdNN model (which demonstrates PR capability), where the updating at time $t+1$ uses the internal state values of any one of the neurons at time $t$.

2. The weight assignment rule for the Mb-AdNN is the same as in M-AdNN and the rationale is the same.

3. The external inputs are applied, in the Mb-AdNN, as in M-AdNN and the rationale is the same..

We now briefly state the stability issues concerning the Mb-AdNN by using Lyapunov Exponents, and then by using the Routh-Hurwitz Criterion.

Theorem 3: The Mb-AdNN described by Equations (2) is locally unstable whenever $N^{1 / 2}>\max \left(1 / k_{r}, 1 / k_{f}\right)$, as demonstrated by its Lyapunov spectrum.

The proof for this theorem can be found in [4.

Remark: There is a value of $m$ for which the maximum between $1 / 2 \ln (N-m+$ $1)+\ln \left(k_{f}\right)$ and $1 / 2 \ln (N-m+1)+\ln \left(k_{f}\right)$ is smaller than 0 . For this value of $m$, the system is will lean more towards behaving like the AdNN, i.e., possessing no chaos because of the absence of positive Lyapunov exponents. For values of $m$ smaller than this critical value, the system is will lean towards behaving like the M-AdNN, because it will exhibit chaos by virtue of the corresponding positive Lyapunov exponents. How $m$ relates to the desynchronization issue is yet unsolved.

Theorem 4: As seen by a Routh-Hurwitz analysis, the necessary conditions for the Mb-AdNN described by Equations (2) to be locally unstable, are that $k_{f}>0$ and $k_{r}>0$.

The proof for this theorem can be found in [4].

We shall now experimentally demonstrate how blurring is achieved as $m$ increases.

\section{$5 \quad$ Experimental Results}

As the reader can observe, a chaotic PR system is distinct with respect to its operation and characteristics, in comparison to more traditional systems. In the training phase, we present the system with a set of patterns, and thus by a sequence of simple assignments (as opposed to a sequence of iterative computations), it "learns" the weights of the CNN. The testing involves detecting a periodicity in the system, signaling the user that a learned pattern has occurred, and then inferring what the periodic pattern is. We shall now demonstrate how the latter task is achieved in a simulation.

In a simulation setting, we are not dealing with a real-life chaotic system (as the brain 2). Indeed, in this case, the output of the CNN is continuously monitored, and a periodic behavior can be observed by studying the frequency

${ }^{2}$ How the brain is be able to record and recognize such a periodic behaviour amidst chaos is yet unexplained [7. 

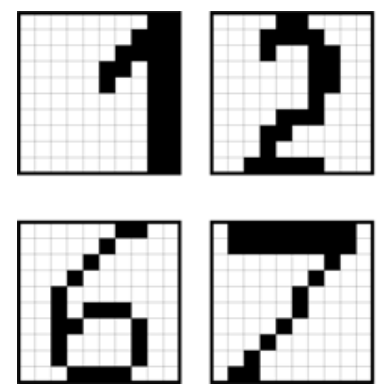
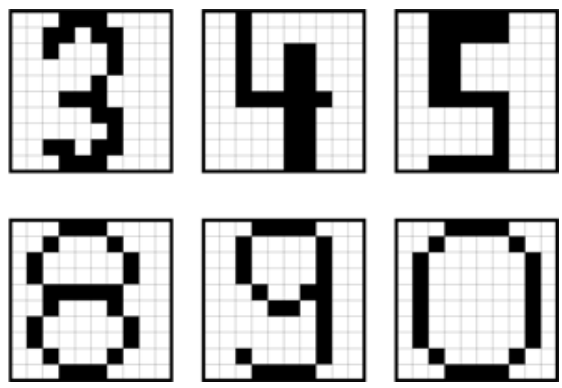

Fig. 1. The set of patterns used in the PR experiments. These were the $10 \times 10$ bitmaps of the numerals $0 \cdots 9$. The initial state used was randomly chosen

spectrum, or by processing the outputs as they come, in the time domain. Notice that the latter is an infeasible task, as the number of distinct outputs could be countably infinite. This is a task that the brain, or, in general, a chaotic system, seems to be able to do, quite easily, and in multiple ways. However, since we have to work with sequential machines, to demonstrate the periodicity, we have opted compare the output patterns with the various trained patterns. Whenever the distance between the output pattern and any trained pattern is less than a predefined threshold, we mark that time instant with a distinct marker characterized by the class of that particular pattern. The question of determining the periodicity of a pattern is now merely one of determining the periodicity of these marker 3 .

We conducted numerous experiments on the Adachi dataset [1] and other datasets. However, in the interest of space, we report the results of training/testing on a numeral dataset described below. The training set had 10 patterns, which consisted of $10 \times 10$ bit-maps of the numerals $0 \cdots 9$ (see Figure 1). The parameters used for Equations (1) were $N=100$ neurons, $\varepsilon=0.00015, k_{f}=0.2$ and $k_{r}=0.9$.

PR Capabilities: The trained M-AdNN, demonstrated a periodic response when non-noisy external stimuli were applied, after an initial non-periodic transient phase. The transient phase was very short - its mean length was 23.1 time units and most of the transitory phases were of length 24 units. The actual length of the transient phase in each case is given in Table 11. The system resonated sympathetically with the input pattern, with a fairly small periodicity.

Blurring Effects: We now examine the "blurring" phenomenon demonstrated by the Mb-AdNN. When $m=1$, the Mb-AdNN degenerates to the M-AdNN

${ }^{3}$ The unique characteristic of such a PR system is that each trained pattern has a unique attractor. When a testing pattern is fed into the system, the system converges to the attractor that best characterizes it. The difficult question, for which we welcome suggestions, is one of knowing which attractor it falls on [3]. 
Table 1. The transitory phase (named "Transient") and the periodicity for Mb-AdNN as the value of $m$ is increased from 1 . The testing samples were the exact non-noisy versions of the original "numerals" training set. Notice that the chaotic PR phenomenon decreases as $m$ increases. It disappears after $m=8$

\begin{tabular}{|c|c|c|c|c|c|c|c|c|c|}
\hline Pattern & & $\mathrm{m}=1$ & $\mathrm{~m}=2$ & $\mathrm{~m}=3$ & $\mathrm{~m}=4$ & $\mathrm{~m}=5$ & $\mathrm{~m}=6$ & $\mathrm{~m}=7$ & $\mathrm{~m}=8$ \\
\hline 1 & Transient & 15 & 15 & $\infty$ & $\infty$ & $\infty$ & $\infty$ & $\infty$ & $\infty$ \\
\hline & Periodicity & 7,15 & 7,15 & $\infty$ & $\infty$ & $\infty$ & $\infty$ & $\infty$ & $\infty$ \\
\hline 2 & Transient & 24 & 24 & 24 & 11 & $\infty$ & $\infty$ & $\infty$ & $\infty$ \\
\hline & Periodicity & 26 & 26 & 25 & 12 & $\infty$ & $\infty$ & $\infty$ & $\infty$ \\
\hline 3 & Transient & 24 & 24 & 24 & 29 & 16 & $\infty$ & $\infty$ & $\infty$ \\
\hline & Periodicity & 26 & 26 & 25 & 25 & 12 & $\infty$ & $\infty$ & $\infty$ \\
\hline 4 & Transient & 24 & 24 & 24 & 11 & $\infty$ & $\infty$ & $\infty$ & $\infty$ \\
\hline & Periodicity & 26 & 26 & 25 & 12 & $\infty$ & $\infty$ & $\infty$ & $\infty$ \\
\hline 5 & Transient & 24 & 24 & 15 & 107 & $\infty$ & $\infty$ & $\infty$ & $\infty$ \\
\hline & Periodicity & 26 & 26 & 7,15 & 120 & $\infty$ & $\infty$ & $\infty$ & $\infty$ \\
\hline 6 & Transient & 24 & 24 & 24 & 11 & $\infty$ & $\infty$ & $\infty$ & $\infty$ \\
\hline & Periodicity & 27 & 27 & 25 & 12 & $\infty$ & $\infty$ & $\infty$ & $\infty$ \\
\hline 7 & Transient & 24 & 24 & 24 & 29 & 31 & 31 & 31 & 42 \\
\hline & Periodicity & 26 & 26 & 25 & 25 & 32 & 32 & 32 & 7,15 \\
\hline 8 & Transient & 24 & 24 & 24 & 29 & 16 & 16 & 16 & $\infty$ \\
\hline & Periodicity & 26 & 26 & 25 & 25 & 12 & 12 & 12 & $\infty$ \\
\hline 9 & Transient & 24 & 24 & 24 & 11 & $\infty$ & $\infty$ & $\infty$ & $\infty$ \\
\hline & Periodicity & 26 & 26 & 26 & 12 & $\infty$ & $\infty$ & $\infty$ & $\infty$ \\
\hline 10 & Transient & 24 & 24 & 24 & 11 & $\infty$ & $\infty$ & $\infty$ & $\infty$ \\
\hline & Periodicity & 26 & 26 & 26 & 12 & $\infty$ & $\infty$ & $\infty$ & $\infty$ \\
\hline \hline
\end{tabular}

leading to a system that exhibits PR, as shown in [3]. Thereafter, as $m$ increases, this PR capabilities decreases in a "smooth" or "gradual" manner when it concerns the number of patterns recognized. But, for the other patterns, the degeneration of the PR capabilities is abrupt - the system "suddenly" changes from being able to recognize a pattern to not being able recognize it.

For $m=2$ (with four eigenvalues different from zero) the Mb-AdNN continued to demonstrates PR capabilities for all the ten patterns. The mean length of the transient phase was 24 time units, and the average periodicity was 23.1 time units. As soon as $m$ was increased to 3 (with six eigenvalues different from zero) the system displayed chaos, but failed to be periodic when teh numeral ' 1 ' is presented. The number of classes for which the periodic behaviour was demonstrated decreases to 3 for $m=5$ and keeps falling till the unity value when $m=8$. For example, when $m=5$, the system recognized only the patterns corresponding to the numerals ' 3 ', ' 7 ' and ' 8 ', and then when $m=7$, the system recognized only the patterns corresponding to the numerals ' 7 ' and ' 8 '. We also emphasize that the periodicity for the same pattern class sometimes changed as the value of $m$ changed. Thus, the periodicity (for $m=7$ ) for the numeral ' 7 ' was 32 time units, and for the numeral ' 8 ' was 12 time units; on the other hand, the periodicity (for $m=8$ ) for ' 7 ' was a double cycle of periodicity 7 and 
15. Observe that the number of patterns for which such a periodic behavior is displayed systematically decreases as $m$ is increased, indicating the phenomenon of increased inaccurate perception or "blurring" even though the input is exact (i.e., non-noisy).

\section{Conclusion}

In this paper, we have studied the problem which is the "inverse" problem of Pattern Recognition (PR), namely that of modeling how even a clear image can be perceived to be blurred in certain contexts. To our knowledge, there is no solution to this in the literature other than for the oversimplified model in which the true image is garbled with noise by the perceiver himself. In this paper we have proposed a chaotic model PR for the theory of "blurring". The paper, which is a extension to a companion paper 3] showed how we can model blurring from the view point of a chaotic PR system. Unlike the companion paper in which the chaotic PR system extracted the pattern from the input, in this case we showed that even without the inclusion of additional noise the perception can be "blurred" if the dynamics of the chaotic system are modified. We thus propose a formal model for chaotic "blurring", and present a rigorous analysis using the Routh-Hurwitz criterion and Lyapunov exponents. We also experimentally demonstrated the validity of our model by using a numeral dataset.

\section{References}

1. Adachi, M., Aihara, K.: Associative Dynamics in a Chaotic Neural Network. Neural Networks 10 (1997) 83-98

2. Buskist, W., Gerbing, D. W.: Psychology: Boundaries and Frontiers. Harpper Collins (1990)

3. Calitoiu, D., Oommen, B.J., Nussbaum, D.: Periodicity and Stability Issues of a Chaotic Pattern Recognition Neural Network. Submitted for Publication. A preliminary version of this paper will be presented at CORES'2005, the 2005 Conference on Computer Recognition Systems, Wroclaw, Poland

4. Calitoiu, D., Oommen, B.J., Nussbaum, D.: Modeling Inaccurate Perception: Desynchronization Issues of a Chaotic Pattern Recognition Neural Network. Submitted for Publication, Unabridged version of this paper (2005)

5. Fausett, L.: Fundamentals of Neural Networks. Prentice Hall (1994)

6. Friedman, F., Kandel, A.: Introduction to Pattern Recognition, statistical, structural, neural and fuzzy logic approaches. World Scientific (1999)

7. Freeman, W.J.: Tutorial in neurobiology: From single neurons to brain chaos. International Journal of Bifurcation and Chaos, 2 (1992) 451-482.

8. Fukunaga, K.: Introduction to Statistical Pattern Recog. Academic Press (1990)

9. Kohonen, T.: Self-Organizing Maps. Springer, Berlin (1997)

10. Piaget, J.: The origins of intelligence in children. International Univ. Press (1952)

11. Ripley, B.: Pattern Recog. and Neural Networks. Cambridge Univ. Press (1996)

12. Schurmann, J: Pattern classification, a unified view of statistical and neural approaches. John Wiley and Sons, New York (1996)

13. Theodoridis, S., Koutroumbas, K.: Pattern recognition. Academic Press (1999). 